\title{
BMJ Open Improving Asthma Care Together (IMPACT) mobile health intervention for school-age children with asthma and their parents: a pilot randomised controlled trial study protocol
}

\author{
Jennifer Sonney (D) , ${ }^{1}$ Teresa Ward (D) , ${ }^{1}$ Hilaire J Thompson (D) , \\ Julie A Kientz (iD , ${ }^{3}$ Chris Segrin (i) ${ }^{4}$
}

To cite: Sonney J, Ward T, Thompson HJ, et al. Improving Asthma Care Together (IMPACT) mobile health intervention for school-age children with asthma and their parents: a pilot randomised controlled trial study protocol. BMJ Open 2022;12:e059791. doi:10.1136/ bmjopen-2021-059791

- Prepublication history and additional supplemental material for this paper are available online. To view these files, please visit the journal online (http://dx.doi.org/10.1136/ bmjopen-2021-059791)

Received 01 December 2021 Accepted 22 December 2021

Check for updates

(C) Author(s) (or their employer(s)) 2022. Re-use permitted under CC BY-NC. No commercial re-use. See rights and permissions. Published by BMJ.

For numbered affiliations see end of article.

Correspondence to Dr Jennifer Sonney; jsonney@uw.edu

\section{ABSTRACT}

Introduction Asthma is an incurable, lifelong condition that places children at increased risk for exacerbation, hospitalisation and school absences. Most paediatric asthma interventions target parents alone and are overly prescriptive. Improving Asthma Care Together (IMPACT) is a novel shared management system comprised of a mobile health (mHealth) application, symptom watch and tailored health intervention that pairs parent and child together as an asthma management team. IMPACT helps families monitor asthma status, tailor asthma management strategies and facilitate intentional transition of asthma management to the child. The purpose of this study is to determine the feasibility, acceptability and preliminary efficacy of the IMPACT intervention.

Methods and analysis This pilot randomised controlled trial will recruit 60 children with asthma (7-11 years) and one parent. All parent-child dyads will complete data collection sessions at baseline, postintervention and follow-up. Dyads randomised to the intervention group (IMPACT) will complete the 8-week intervention comprised of weekly activities including symptom monitoring, goal setting and progress monitoring. Dyads randomised to the control group will receive usual care but then be provided access to IMPACT at the end of the study. Feasibility will be measured by the proportion of eligible dyads enrolled and retained. Acceptability of IMPACT will be assessed using the Acceptability of Intervention Measure, the System Usability Scale and a semistructured interview. Preliminary efficacy is determined based on change in primary outcomes, parent-reported and child-reported asthma responsibility and asthma self-efficacy scores, from baseline.

Ethics and dissemination This study has been approved by the University of Washington Institutional Review Board; study ID: STUDY00010461. Participants gave informed consent to participate in the study before taking part. Study results will be disseminated in peer-reviewed journals and scientific conferences. A lay summary will be provided to study participants.

Trial registration number NCT04908384 (ClinicalTrials. gov identifier).

\section{Strengths and limitations of this study}

- The Improving Asthma Care Together (IMPACT) mobile health intervention was iteratively codesigned by its intended end users, children with asthma and their parents.

- The dyadic study design pairs parents and children together to learn to share asthma management together.

- The study protocol includes remote study visit opportunities to broaden access to the IMPACT intervention.

- Given the pilot nature of this study, the IMPACT intervention is available only in English.

- The same size is small, which will limit generalisability of study findings.

\section{INTRODUCTION}

Asthma is most common chronic condition of childhood, affecting over six million US children. ${ }^{1}$ Asthma is an incurable, lifelong condition that places children at increased risk for functional impairments, decreased quality of life, school absences, increased healthcare utilisation and irreversible structural airway remodelling. ${ }^{2-5}$ Asthma management requires symptom monitoring and response, trigger avoidance and timely and appropriate medication use. ${ }^{256}$ Unfortunately, fewer than $50 \%$ of children with asthma are adherent to management regimens, leading to increased disease morbidity and mortality and potentially irreversible airway damage. ${ }^{37}$ Improving paediatric asthma management represents a critical health need.

The school-age years (7-11) represent a natural transition in asthma management, as children assume increasing responsibility for asthma-related care while they progressively spend more time away from parents 
at school and other extracurricular activities. ${ }^{8}$ Developmentally, school-age children are rule-driven, understand right and wrong and are able to problem solve, which supports their capability to be active participants in their own asthma management. ${ }^{9-15}$ Yet, the majority of existing interventions focus on parents alone and use prescriptive approaches, telling the parent what to 'do' to the child to manage their asthma. ${ }^{16-21}$ As a result, our current strategies are failing to provide children with asthma and their families the tools they need to manage asthma together within the realities of their daily lives. ${ }^{12} 13$ The answer to this problem lies in shared asthma management by the parent-child dyad, ${ }^{12} 13$ with the school-age years the ideal developmental period for children to begin sharing responsibility with their parents and establishing lifelong health behaviours. ${ }^{22-25}$

Using a Human-Centred Design framework, we collaborated with parent-child dyads to codesign Improving Asthma Care Together (IMPACT), a tailored shared management mobile health (mHealth) application that pairs the parent and child together as a shared management team. ${ }^{26}{ }^{27}$ IMPACT aims to help families tailor asthma management strategies to fit the realities of their social environments while facilitating intentional transition of asthma management to the child. We hypothesise that by giving children a voice in their own care, we will not only improve asthma management in the present but also support the successful future transition of asthma management to those individuals who will ultimately assume sole responsibility in managing their lifelong condition. The purpose of this pilot-randomised controlled trial is to determine the feasibility, acceptability and initial efficacy of IMPACT.

\section{METHODS AND ANALYSIS}

\section{Study design and setting}

This study protocol was developed in alignment with the Standard Protocol Items: Recommendations for Interventional Trials guidelines. ${ }^{28}$ We will use a parallel group, randomised controlled pretest-posttest design to compare the 8-week IMPACT intervention with a usual care control. Data will be collected at baseline (T0), postintervention (T1) and 8-week follow-up (T2). See figure 1 for the schedule of enrolment, intervention and assessment. The study will take place within participant homes, with data collection visits occurring either in person (if within the greater Seattle, Washington area) or remotely via Zoom videoconference (Zoom Video Communications, San Jose, California). Any trial modifications will be approved by the Institutional Review Board prior to implementation and will be reported to the trial registry.

\section{Participants}

Sixty children and one parent (or caregiver) dyad will be recruited for this study. Child eligibility criteria include: (1) age 7-11 years, (2) diagnosed with persistent asthma (defined as a prescription for daily asthma medication) and (3) speak English. Parent eligibility criteria include: (1) child's primary caregiver residing with the child $50 \%$ or more, (2) 18 years or older, (3) speak and read English, (4) have a smartphone with data plan and (5) have a parent-reported asthma responsibility mean score of $\leq 2.5$ (indicating parent assumes majority of asthma management responsibility). The study catchment area is the USA. Participants who reside in the greater Seattle area in Washington State will have the option for in person visits, whereas those who reside outside of the greater Seattle area will be eligible for remote visits only. Children will be excluded if they have parent-reported conditions that may impair the child's ability to learn shared management, including developmental delay (language $<5$ year level), comorbid condition (cancer, diabetes, autism spectrum disorder) or current asthma exacerbation at the time of recruitment (defined as a prescription for oral corticosteroids), as this is a serious health threat and not an opportune time for learning shared management. There are no parental exclusion criteria.

\section{Recruitment, screening and consent}

Numerous strategies will be used to recruit the study sample, including social media posts, elementary school flyers and recruitment flyers posted in community-based paediatric clinics and other community locations (eg, libraries). Prospective participants will also be identified through an electronic medical record screening within UW Medicine, a large hospital system and network of neighbourhood clinics in Washington State, and receive a study flyer via mail or email. All recruitment materials will contain the study screening weblink and quick response $(\mathrm{QR})$ code as well as the Principal Investigator (PI) contact information.

REDCap, a secure, encrypted online data management system, ${ }^{29}$ will be used for all study-related data collection and management, including study screening. The IMPACT weblink and QR codes will direct users to the IMPACT REDCap webpage and prompt them to complete the eligibility screening. The screening will be automatically scored and indicate whether participants appear to meet eligibility criteria or not. Those who are not eligible will be thanked for their time. Those who are eligible will be asked to provide their contact information in order for the study team to contact them. They will then access the e-consent form (online supplemental file 1), with electronic signature enabled as well as a video assent form for children. The study team will contact all eligible participants to answer any questions, discuss consent and assent (including offer of paper forms if preferred) and to schedule a baseline data collection session.

\section{Data collection}

Data collection sessions will occur either in person or remote via Zoom videoconference. Participants who live within the greater Seattle, Washington area will be given the choice between remote or in person visits. 


\begin{tabular}{|c|c|c|c|c|c|}
\hline & \multicolumn{5}{|c|}{ STUDY PERIOD } \\
\hline & \multirow[t]{2}{*}{ Enrollment } & \multirow{2}{*}{$\frac{\text { Allocation }}{\text { T0 }}$} & \multicolumn{2}{|c|}{ Post-Allocation } & \multirow{2}{*}{$\frac{\text { Closeout }}{\mathrm{T} 2}$} \\
\hline Timepoint & & & 8 Weeks & T1 & \\
\hline \multirow{2}{*}{$\begin{array}{l}\text { ENROLLMENT: } \\
\text { Eligibility Screening }\end{array}$} & & & & & \\
\hline & $x$ & & & & \\
\hline Informed consent (P) & $x$ & & & & \\
\hline \multirow{2}{*}{$\begin{array}{l}\text { Assent (C) } \\
\text { Allocation }\end{array}$} & $X$ & & & & \\
\hline & & $X$ & & & \\
\hline \multirow{3}{*}{$\begin{array}{l}\text { INTERVENTION: } \\
\text { IMPACT } \\
\text { Control group }\end{array}$} & & & & & \\
\hline & & $\diamond$ & $\longrightarrow$ & & \\
\hline & & $\hookleftarrow$ & $\longrightarrow$ & & \\
\hline \multirow{3}{*}{$\begin{array}{l}\text { ASSESSMENTS: } \\
\text { Demographics }(P) \\
\text { Asthma responsibility }(P, C)\end{array}$} & & & & & \\
\hline & & $\mathrm{X}$ & & & \\
\hline & & $x$ & & $x$ & $x$ \\
\hline \multirow{2}{*}{$\begin{array}{l}\text { Asthma self-efficacy }(P, C) \\
\text { Asthma control test }(P, C)\end{array}$} & & $X$ & & $X$ & $X$ \\
\hline & & $X$ & & $\mathrm{X}$ & $X$ \\
\hline Lung functioning/Spirometry (C) & & $X$ & & $X$ & $X$ \\
\hline \multirow{2}{*}{$\begin{array}{l}\text { Medication adherence }(P, C) \\
\text { Asthma } Q O L(P, C)\end{array}$} & & $X$ & & $x$ & $x$ \\
\hline & & $X$ & & $x$ & $x$ \\
\hline Family functioning (P) & & $x$ & & $x$ & $x$ \\
\hline \multirow{2}{*}{$\begin{array}{l}\text { Medication beliefs }(P, C) \\
\text { Illness perception }(P, C)\end{array}$} & & $x$ & & $X$ & $X$ \\
\hline & & $x$ & & $x$ & $x$ \\
\hline Feasibility of intervention $(P, C)$ & & & & I & \\
\hline \multirow{2}{*}{$\begin{array}{l}\text { Acceptability of intervention }(P, C) \\
\text { System usability }(P, C)\end{array}$} & & & & I & \\
\hline & & & & I & \\
\hline Semi-structured interview $(P, C)$ & & & & 1 & \\
\hline
\end{tabular}

Figure 1 SPIRIT Schedule of enrolment, interventions and assessment. C, child; I, intervention group only; P, parent; QOL, quality of life; SPIRIT, Standard Protocol Items: Recommendations for Interventional Trial; X, all participants.

Participants outside of the greater Seattle area will only be offered remote visits. Data collection will occur via REDCap. Participants who withdraw during the study will be encouraged to complete data collection sessions and reasons for withdrawal will be documented.

\section{Baseline (T0)}

\section{In person procedures}

The study team will perform direct child measures (height and weight). Next, the study team will assist the parent with pairing the spirometer with their mobile device. Note, spirometers use a reference database to evaluate lung functioning. As such, the spirometer requires patient details including age, gender, height and weight. The study team will instruct the parent to input the study identification number (ID) instead of the child's name and year of birth (not date) to avoid unnecessary personal health information disclosure. The study team will coach the child through spirometry and upload a screenshot of the results to the REDCap database. Note, every effort will be made to perform spirometry outside (weather permitting) and remain socially distanced while wearing masks, given that spirometry is considered an aerosolising procedure.

Next, individual data collection will commence. The parent participants will be provided an iPad with the electronic REDCap instruments preloaded. Parents will be instructed to complete the instruments to the best of their ability and to ask questions as needed. At the same time, the study team will work 1:1 with the child to assist with instrument completion using a separate iPad (reading items if requested). Should the parent finish their instruments prior to the child, the parent will be asked to refrain from providing input on the child's answers. The estimated time burden for data collection is less than 1 hour. Following data collection, the study team will randomise parent-child dyads into intervention 
or control groups (described below). For those assigned to the intervention group, the study personnel will assist intervention dyads with downloading the IMPACT app, pairing the symptom watch and reader with app and reviewing written instructions for use. Dyads assigned to the control arm will receive an information sheet about upcoming data collection sessions and be advised keep the spirometer for future study sessions. Participant dyads will be emailed a US\$50 digital gift card following the T0 visit.

\section{Remote procedures}

To facilitate a remote baseline visit, the study team will mail the study supplies to participants prior to the videoconference study visit. During the remote visit, the study team will assist the parent with pairing the spirometer with their mobile device and coach the child through performing spirometry. Parents will be asked to email the spirometry report to the study team. Next, the study team will send the parent a personalised weblink to complete their study instruments via REDCap (either using a different device or after the visit). The study team will screenshare the child's REDCap study instruments and assist with completion, specifically asking that the parent not interfere with the child's answers. Children may choose to verbalise their answers or use Zoom 'mouse control' to self-select their responses. Randomisation and instructions, based on group assignment, and compensation will follow the procedure described above.

\section{Postintervention (T1)}

All parent-child dyads will complete a $\mathrm{T} 1$ postintervention (8weeks) study visit, either in person or remote. Survey completion procedures will mirror the T0 protocol. Additionally, families assigned to the intervention group will complete a brief semistructured interview to assess the acceptability of the intervention. Participant dyads will be emailed a US $\$ 100$ digital gift card following the T1 visit.

\section{Follow-up (T2)}

All parent-child dyads will complete a T2 follow-up study visit 8 weeks after T1, either in person or remote. Survey completion will follow the previously described protocol. For control group families that are interested, the study team will assist with accessing the IMPACT app and orient the dyad to app use. Participant dyads will be emailed a $\$ 150$ digital gift card following the T2 visit.

\section{Randomisation}

A statistician independent from the study team will generate a block randomisation allocation sequence, stratified by child gender. The order of the blocks will be random. This sequence will be uploaded to the REDCap IMPACT data management system. Following the T0 data collection, the REDCap randomisation module will automatically assign parent-child dyads to the intervention or control group. The study statistician will be blinded to participant allocation.

\section{Sample size}

Power estimates are based on projected sample of 30 per arm, assuming $15 \%$ attrition. A sample of 60 will provide $80 \%$ power to detect an effect as small as $d=0.28$ in a paired t test (eg, child spirometry) or a parent-child correlation as small as $r=0.22$. Statistical power of dyadic analyses based on the Actor-Partner Inter-dependence Model, ${ }^{30}$ appropriate for variables such as self-efficacy and quality of life, was estimated with the APIMPowerRdis ${ }^{31}$ routine in $\mathrm{R}$, assuming modest actor effects with a partial $r=0.35$, partner effects with a partial $r=0.20$ and correlation of the parent-child T0 variables and error terms of $r=0.35$. Such a model would have power $=0.98$ to detect the actor effects and 0.67 to detect the partner effects. With 30 cases in each group, mixed model analyses of covariance (ANCOVAs) that compare the groups at two time points, assuming an average correlation of $r=0.60$ between measures, would have power $=0.80$ to detect group difference effects as small as $\mathrm{f}=0.28\left(\eta^{2}=0.05\right)$, and power $=0.80$ to detect the within groups pre-/postdifference as small as $\mathrm{f}=0.17\left(\eta^{2}=0.02\right)$ and a Group $\times$ Time interaction (the most critical of the three effects) as small as $\mathrm{f}=0.37\left(\eta^{2}=0.07\right)$.

\section{Measures}

\section{Participant characteristics}

Parents will complete an investigator-developed survey, including demographic information about the parent and child. Parent characteristics include gender identity, education, employment status, race and ethnicity. Child characteristics include age, gender identity, sex at birth (necessary for spirometry algorithm), grade in school, race, ethnicity and insurance coverage. The survey also includes a brief asthma history, including age at asthma diagnosis, number of asthma attacks (exacerbations) in past year, steroid prescriptions in past year and history of asthma emergency department visits, hospitalisation and/or intensive care admission.

\section{Primary outcome measures}

Asthma responsibility

The Asthma Responsibility Questionnaire (ARQ) is comprised of 10 items using a 5-point scale to report asthma management task responsibility, with higher scores indicating higher management responsibility for the child. ${ }^{32}$ The ARQ has established reliability and validity. ${ }^{32}$

\section{Asthma self-efficacy}

Asthma Management Self-Efficacy scale (13-items for parent, 12-items for child) uses a 5-point scale to assess asthma self-efficacy, with higher scores indicating higher self-efficacy. ${ }^{33}$ The Asthma Management Self-Efficacy scale has established reliability and validity. ${ }^{33}$

Secondary outcome measures

Asthma control

Asthma control will be evaluated using a self-report scale, the Childhood Asthma Control Test (C-ACT). ${ }^{34}$ 
The C-ACT is a clinically validated 7-item scale, with three items for parents (6-point scale) and four for children (4-point scale), to assess asthma control. Scores are summed, with higher scores indicating better asthma control. Clinically established cut points will be used to classify asthma status, with scores $\leq 12$, indicating poorly controlled asthma, 13-19, not well-controlled asthma, and $\geq 20$ well controlled asthma. ${ }^{3435}$

\section{Lung functioning}

Spirometry, an objective measure of lung functioning, is a standard clinical tool to assess lung functioning. The Spirobank Smart is a single-patient reusable handheld spirometer that complies with the American Thoracic Society and European Respiratory Society standards for spirometry as outlined in the International Organization for Standardization (MIR Medical International Research, Rome, Italy). The Spirobank Smart pairs via Bluetooth to the companion Spirobank health app provides instructional prompts, visual incentives and generates spirometry performance reports based on the Global Lung Initiative reference population. Using the Spirobank Smart and companion app, all child subjects will perform spirometry following coaching from the study team. Asthma control will be assessed based on two spirometry values calculated by the device: the forced expiratory volume in 1s (FEV1) and FEV1/Forced Vital Capacity (FVC) ratio. Asthma control classification as well controlled, not well controlled or very poorly controlled will be based on the NHLBI clinical asthma guideline control cut points FEV1 and FEV1/FVC. ${ }^{36}$ There is potential disagreement between C-ACT and spirometry values; should this occur, the study team will prioritise the objective measure (spirometry) over the C-ACT score.

\section{Medication adherence}

The Medication Adherence Report Scale for Asthma (MARS-A) is comprised of 10 items using a 5-point scale to assess reported asthma controller medication adherence. ${ }^{37} \mathrm{~A}$ mean score of $\geq 4.5$ indicates adherence. While the MARS-A has established reliability and validity in adults; ${ }^{37}$ to our knowledge, it has been used, though not validated, in previous studies with children and adolescents. $^{38-40}$

\section{Asthma-related quality of life}

The Mini Asthma Quality of Life Questionnaire (child) ${ }^{41}$ and Pediatric Asthma Caregiver Quality of Life Questionnaire (parent) ${ }^{42}$ are designed to measure the physical, social and emotional impact of asthma on one's life. Both scales are comprised of 13 items using a 7-point scale, with higher scores indicating higher quality of life. Both scales have established reliability and validity. ${ }^{41} 42$

\section{Exploratory outcome measures}

\section{Family functioning}

The McMaster Family Assessment Device (FAD) is a 60-item parent-report instrument that uses a 4-point scale to evaluate family functioning. ${ }^{43}$ Higher scores indicate worsened levels of family functioning. The FAD has established reliability and validity in families with school-age children. ${ }^{44}$

\section{IIIness perception}

The Brief Illness Perception Questionnaire (BIPQ) is an 8-item scale that uses a 10-point scale to assess individual beliefs about a health condition. ${ }^{45}$ Higher scores indicate more burdensome perception. The BIPQ has established reliability and validity in adults ${ }^{45}$ and children. ${ }^{46}$

\section{Medication beliefs}

The Beliefs about Medicines Questionnaire (BMQ) uses a 5-point scale to assess perceived medication necessity (5 items) and concerns (5 items). ${ }^{47}$ The perceived necessityconcern differential is calculated by subtracting the sum of the concern item scores from the sum of the necessity item scores. A positive necessity-concern differential indicates perceived medication necessity outweighs concerns, whereas a negative differential indicates that perceived medication concerns outweigh perceived necessity. The $\mathrm{BMQ}$ has established validity and reliability in adults ${ }^{47}$ as well as parents and children. ${ }^{48}$

\section{Impact feasibility and acceptability Feasibility}

The feasibility of IMPACT will be determined by study eligibility, enrolment, retention and use of the IMPACT intervention. We will also use the Feasibility of Intervention measure, a 4-item scale that uses a 5-point scale, with higher scores indicating higher intervention feasibility. ${ }^{49}$

\section{Acceptability (intervention group only)}

Acceptability of IMPACT will be assessed using the Acceptability of Intervention Measure (AIM), System Usability Scale (SUS) and semistructured interview. ${ }^{49} 50$ The AIM is comprised of 4 items and uses a 5-point scale, with higher scores indicating higher acceptability. The SUS is a 10-item scale that uses a 5-point scale to report the perceived usability of a system, with higher scores indicating higher usability. Both scales have established reliability and validity. ${ }^{49}{ }^{50} \mathrm{~A}$ brief, investigator-developed 12-question semistructured interview will be used to elicit parent and child feedback on the IMPACT intervention.

\section{Impact intervention}

The IMPACT system was designed to facilitate parentchild asthma monitoring as well as improved shared management of asthma responsibility. The IMPACT system is comprised of a wearable 'symptom watch', spirometer and mHealth IMPACT app. The symptom watch is worn by the child and functions as an event marker to allow children to report asthma symptoms as they occur. These symptom events are synced with the IMPACT app and tracked graphically within the IMPACT dashboard. The dashboard also tracks weekly asthma control scores (C-ACT) as well as child spirometry values (FEV1). 
The IMPACT intervention is imbedded within the IMPACT app. Parent-child dyads will be prompted by push notification to complete weekly study activities throughout the 8-week intervention. Each week, dyads will be prompted to complete the C-ACT and child spirometry. Next, dyads will be prompted to select a weekly evidence-based asthma shared management goal, such as conducting asthma check-ins together to discuss symptoms and medication use or remembering to take daily asthma medication doses. After selecting goals, participants will be prompted to anticipate barriers and problem solve those barriers together. Each subsequent week, participants will also be prompted to evaluate their progress together.

\section{Control group}

Dyads assigned to the control group will receive usual asthma care from their healthcare provider(s). Additionally, the principal investigator will conduct an asthma control assessment, based on the C-ACT and spirometry, and provide an asthma resource list. This report may be shared with the child's healthcare provider(s), if desired.

\section{Statistical analyses}

\section{Feasibility}

Eligibility, enrolment and retention (number who complete the study) data will be summarised in a study flowchart. A priori feasibility benchmarks include $\geq 60 \%$ recruitment (eligible dyads who enrol) and $\geq 80 \%$ retention. Study withdrawal reasons will also be reported.

\section{Acceptability}

Participants will report on IMPACT acceptability via the AIM and SUS scales as well as by semistructured interview. The a priori acceptability benchmark will be $\geq 60 \%$ of participants rating IMPACT as acceptable ( 4 or 5 on AIM, 7-10 on SYS). Semistructured interview data will undergo thematic analysis to identify, analyse and report patterns within the data. Preliminary codes will be generated, then the study team will work together to code the data with existing, refined or newly created codes and then identify themes. After this, the team will define and name each theme.

\section{Primary outcomes (asthma responsibility score, self-efficacy)}

Descriptive summaries of deidentified data will be transferred into SPSS V.24 (IBM, Armonk, New York) for analyses. Distributions of interval and ratio level variables will be checked for normality and transformed as necessary. We will compare characteristics of those who completed the study to those who did not to inform the generalisability of findings. We expect an increase in asthma management responsibility scores and increased dyadic interdependence (ie, non-independence of scores between dyads) ${ }^{30}$ between parent and child-reported outcomes over time in the intervention group, suggesting true shared management.

\section{Secondary outcomes}

Secondary outcome analyses will use mixed model ANCOVAs to assess whether the primary outcome variable means, adjusted for baseline scores, differ between intervention and control groups. We predict a Group $\times$ Time interaction such that shared management and health outcome variables (asthma responsibility score, self-efficacy, medication adherence, asthma control, quality of life (QOL)) will increase to a greater extent in the intervention group. The dyadic effect of the intervention will be tested with the Actor-Partner Interdependence Model $^{3051}$ using structural equation modelling with full information maximum likelihood (FIML) estimation. First, the pattern of missing data will be tested to determine whether it is missing at random. The FIML method is superior to listwise deletion of missing data points in terms of producing less biased estimates. Next, intraclass correlations will be examined for parent and child asthma responsibility, self-efficacy,and general health scores. The APIM produces estimates of actor effects (Person A's pretest $\rightarrow$ Person A's post-test) and partner effects (Person A's pretest $\rightarrow$ Person B's post-test). Actor effects are estimated while controlling for partner effects, and vice versa. In these analyses, dyadic interdependence is indicated by statistically significant partner effects (eg, parent pretest $\rightarrow$ child post-test, controlling for child pretest). Intervention condition will be entered into the model as a dummy-coded variable. For each measure taken from both parent and child (ie, self-efficacy, asthma responsibility), two APIMs will be compared; one in which all of the paths from child variables are constrained equal to their respective paths from the parent variables, and one in which all paths are unconstrained and, thus, free to vary. Comparison of the fit of these two models indicates whether the effects emanating from the parent are significantly different from those emanating from the child. Parent-child dyads in IMPACT are expected to show stronger partner effects than those in the comparison group.

\section{Family and public involvement}

The IMPACT system and intervention were iteratively codesigned by children with asthma and their parents. Generative research determined parent-child dyad needs and priorities with respect to asthma management. ${ }^{27}$ Next, dyads worked alongside the study team to select the type of intervention (mobile health app and wearable) and refine the features to ensure they address dyad-identified priorities. ${ }^{26}{ }^{27}$ Finally, dyads participated in extensive usability testing and refinement to ensure IMPACT emerged as an engaging, useful and functional system to support shared asthma management. ${ }^{26}$ Parent-child dyads will not be involved in participant recruitment for this pilot RCT, but study results will be disseminated to all families that contributed to the development of IMPACT.

\section{ETHICS AND DISSEMINATION Data management}

All appropriate steps will be taken to maintain security of participant data. All participants will be assigned a unique study identifier. Electronic study records will be stored within the REDCap database, a secure cloud-based data management system that is compliant with the Health Insurance Portability and Accountability Act. IMPACT 
intervention use data will be stored in an encrypted cloud database. Only the study team members will have access to the final study data set. Data quality will be ensured by having survey completions verified within REDCap. Ongoing quality control procedures will be implemented, to include an audit of selected cases (10\%) to ensure adherence with IRB requirements and study protocols.

\section{Participant safety}

Given that this study was deemed 'minimal risk', we do not anticipate any serious adverse events. However, to ensure participant safety, we will send a monthly report of all unanticipated problems, adverse events and protocol deviations to a Scientific Monitoring Committee (SMC), comprised of a paediatric pulmonologist and senior nurse scientist. Potential adverse events will be graded by the PI and SMC following standard procedures for the University of Washington Adverse Event Reporting Policy. Any potential serious adverse events, such as those resulting in medical problems or breach of confidentiality, will be reviewed by PI and SMC, graded and reported to the Institutional Review Board.

\section{Ethics approval and consent to participate}

The University of Washington Institutional Review Board approved this protocol and deemed the study minimal risk. We will submit any protocol modifications to the Institutional Review Board for approval; once approved, the ClinicalTrials.gov registry will be subsequently updated. Study participants will complete consent (parent) and assent (child) procedures, as described above.

\section{Dissemination}

Aggregated study findings will be disseminated via publication and presentation to paediatric clinical, scientific and clinical informatics audiences. In addition, we will provide study results to our community of interest, specifically parent-child dyads that assisted with IMPACT development and testing. Authorship eligibility will follow the International Committee of Medical Journal Editors recommendations. ${ }^{52}$ Findings from this study will inform future refinements of the IMPACT system and, if the preliminary evidence is promising, a full-scale clinical trial with broader participant representation. We also anticipate that our study may serve as a pragmatic example of designing behavioural interventions to promote parentchild shared management of chronic health conditions.

\section{Study status}

Screening and recruitment commenced on 1 October 2021. This study is ongoing until July 2022 (estimated).

\footnotetext{
Author affiliations

${ }^{1}$ Child, Family, and Population Health Nursing, University of Washington School of Nursing, Seattle, Washington, USA

${ }^{2}$ Biobehavioral Nursing and Health Informatics, University of Washington School of Nursing, Seattle, Washington, USA

${ }^{3}$ Human Centered Design \& Engineering, University of Washington Seattle Campus, Seattle, Washington, USA

${ }^{4}$ Department of Communication, The University of Arizona, Tucson, Arizona, USA
}

Contributors JS, TW, HJT, JAK and CS conceived the study and study design. JS drafted the study protocol. All authors were involved in editing the protocol for critically important context and approved the final version.

Funding This work was supported by the National Institutes of Health/National Institute of Nursing Research grant number R21NR019328 and the National Institutes of Health/National Center for Advancing Translational Sciences KL2TR002317. JS also receives support from the US Health Resources Services Administration grant number T72MC00007. The content is solely the responsibility of the authors and does not necessarily reflect the official views of the funding source, nor will the funding source have any role in the design, execution, analysis, interpretation or dissemination of the study.

Competing interests None declared.

Patient consent for publication Not applicable.

Provenance and peer review Not commissioned; peer reviewed for ethical and funding approval prior to submission.

Supplemental material This content has been supplied by the author(s). It has not been vetted by BMJ Publishing Group Limited (BMJ) and may not have been peer-reviewed. Any opinions or recommendations discussed are solely those of the author(s) and are not endorsed by BMJ. BMJ disclaims all liability and responsibility arising from any reliance placed on the content. Where the content includes any translated material, BMJ does not warrant the accuracy and reliability of the translations (including but not limited to local regulations, clinical guidelines, terminology, drug names and drug dosages), and is not responsible for any error and/or omissions arising from translation and adaptation or otherwise.

Open access This is an open access article distributed in accordance with the Creative Commons Attribution Non Commercial (CC BY-NC 4.0) license, which permits others to distribute, remix, adapt, build upon this work non-commercially, and license their derivative works on different terms, provided the original work is properly cited, appropriate credit is given, any changes made indicated, and the use is non-commercial. See: http://creativecommons.org/licenses/by-nc/4.0/.

\section{ORCID iDs}

Jennifer Sonney http://orcid.org/0000-0002-0766-2918

Teresa Ward http://orcid.org/0000-0002-8651-4066

Hilaire J Thompson http://orcid.org/0000-0002-5472-478X

Julie A Kientz http://orcid.org/0000-0001-7437-7861

Chris Segrin http://orcid.org/0000-0003-1797-2503

\section{REFERENCES}

1 Centers for Disease Control and Prevention. Most recent national asthma data Atlanta. GA: US Department of Health and Human Services, 2019. https://www.cdc.gov/asthma/most_recent_national_ asthma data.htm

2 National asthma education prevention program (NAEPP). Expert panel report 3 (EPR-3): guidelines for the diagnosis and management of Asthma-Summary report 2007. J Allergy Clin Immunol 2007;120:S94-138.

3 Celano MP, Linzer JF, Demi A, et al. Treatment adherence among low-income, African American children with persistent asthma. $J$ Asthma 2010;47:317-22.

4 Grainge CL, Lau LCK, Ward JA, et al. Effect of bronchoconstriction on airway remodeling in asthma. N Engl J Med 2011;364:2006-15.

5 Brand PLP, Mäkelä MJ, Szefler SJ, et al. Monitoring asthma in childhood: symptoms, exacerbations and quality of life. Eur Respir Rev 2015;24:187-93.

6 McQuaid EL, Kopel SJ, Klein RB, et al. Medication adherence in pediatric asthma: reasoning, responsibility, and behavior. $J$ Pediatr Psychol 2003;28:323-33.

7 Elkout H, Helms PJ, Simpson CR, et al. Adequate levels of adherence with controller medication is associated with increased use of rescue medication in asthmatic children. PLoS One 2012;7:e39130-6.

8 Buford TA. School-Age children with asthma and their parents: relationships with health care providers. Issues Compr Pediatr Nurs 2005;28:153-62.

9 Olson LM, Radecki L, Frintner MP, et al. At what age can children report dependably on their asthma health status? Pediatrics 2007;119:e93-102.

10 Rebok G, Riley A, Forrest C, et al. Elementary school-aged children's reports of their health: a cognitive interviewing study. Qual Life Res 2001;10:59-70. 
11 Riley AW. Evidence that school-age children can self-report on their health. Ambul Pediatr 2004;4:371-6.

12 Kieckhefer GM, Trahms CM. Supporting development of children with chronic conditions: from compliance toward shared management. Pediatr Nurs 2000;26:354-63.

13 Sonney JT, Insel KC. Reformulating the common sense model of self-regulation: toward parent-child shared regulation. Nurs Sci Q 2016;29:154-9.

14 Malerstein AJ, Ahern MM. Piaget's stages of cognitive development and adult character structure. Am J Psychother 1979;33:107-18.

15 Newton JT, Harrison V. The cognitive and social development of the child. Dent Update 2005;32:33-8.

16 Marcano Belisario JS, Huckvale K, Greenfield G, et al. Smartphone and tablet self management apps for asthma. Cochrane Database Syst Rev 2013:11:CD010013.

17 Bender BG, Cvietusa PJ, Goodrich GK, et al. Pragmatic trial of health care technologies to improve adherence to pediatric asthma treatment: a randomized clinical trial. JAMA Pediatr 2015;169:317-23.

18 Vasbinder EC, Janssens HM, Rutten-van Mölken MPMH, et al. e-Monitoring of asthma therapy to improve compliance in children using a real-time medication monitoring system (RTMM): the eMATIC study protocol. BMC Med Inform Decis Mak 2013;13:38.

19 Welsh EJ, Hasan M, Li P. Home-based educational interventions for children with asthma. Cochrane Database Syst Rev 2011;10:CD008469.

20 Shegog R, Bartholomew LK, Parcel GS, et al. Impact of a computerassisted education program on factors related to asthma selfmanagement behavior. J Am Med Inform Assoc 2001;8:49-61.

21 Chiang L-C, Huang J-L, Yeh K-W, et al. Effects of a self-management asthma educational program in Taiwan based on PRECEDEPROCEED model for parents with asthmatic children. J Asthma 2004:41:205-15.

22 Ekim A, Ocakci AF. Perceptions of parents and children regarding asthma management responsibilities. J Spec Pediatr Nurs 2013;18:289-96.

23 Sonney JT, Gerald LB, Insel KC. Parent and child asthma illness representations: a systematic review. J Asthma 2016;53:510-6.

24 Butz AM, Walker JM, Pulsifer M, et al. Shared decision making in school age children with asthma. Pediatr Nurs 2007;33:111-6.

25 Brown N, Gallagher R, Fowler C, et al. The role of parents in managing asthma in middle childhood: an important consideration in chronic care. Collegian 2010;17:71-6.

26 Sonney J, Cho Emily (Enubi), Zheng Q, et al. Refinement of a parentchild shared asthma management mHealth APP: human centered design study (Preprint). JMIR Pediatr Parent 2021.

27 Sonney J, Duffy M, Hoogerheyde LX, et al. Applying HumanCentered design to the development of an asthma essentials kit for school-aged children and their parents. J Pediatr Health Care 2019;33:169-77.

28 Chan A-W, Tetzlaff JM, Altman DG, et al. SPIRIT 2013 statement: defining standard protocol items for clinical trials. Ann Intern Med 2013:158:200-7.

29 Harris PA, Taylor R, Thielke R, et al. Research electronic data capture (REDCap)--a metadata-driven methodology and workflow process for providing translational research informatics support. J Biomed Inform 2009;42:377-81.

30 Kenny DA, Kashy DA, Cook WL. Dyadic data analysis. New York, NY: The Guildford Press, 2006.

31 Ackerman RA, Kenny DA. APIMPowerR: An interactive tool for Actor-Partner Interdependence Model power analysis [computer software], 2016. Available: https://robert-a-ackerman.shinyapps.io/ APIMPowerRdis/ [Accessed 25 Apr 2017].

32 McQuaid EL, Penza-Clyve SM, Nassau JH, et al. The asthma responsibility questionnaire: patterns of family responsibility for asthma management. Children's Health Care 2001;30:183-99.

33 Bursch B, Schwankovsky L, Gilbert J, et al. Construction and validation of four childhood asthma self-management scales: parent barriers, child and parent self-efficacy, and parent belief in treatment efficacy. Journal of Asthma 1999;36:115-28.

34 Liu AH, Zeiger R, Sorkness C, et al. Development and crosssectional validation of the childhood asthma control test. J Allergy Clin Immunol 2007;119:817-25.

35 Liu AH, Zeiger RS, Sorkness CA, et al. The childhood asthma control test: retrospective determination and clinical validation of a cut point to identify children with very poorly controlled asthma. J Allergy Clin Immunol 2010;126:e1:267-73.

36 Expert Panel Working Group of the National Heart, Lung, and Blood Institute (NHLBI) administered and coordinated National Asthma Education and Prevention Program Coordinating Committee (NAEPPCC), Cloutier MM, Baptist AP, et al. 2020 Focused Updates to the Asthma Management Guidelines: A Report from the National Asthma Education and Prevention Program Coordinating Committee Expert Panel Working Group. J Allergy Clin Immunol 2020;146:1217-70.

37 Cohen JL, Mann DM, Wisnivesky JP, et al. Assessing the validity of self-reported medication adherence among inner-city asthmatic adults: the medication adherence report scale for asthma. Ann Allergy Asthma Immunol 2009;103:325-31.

38 Sonney J, Insel KC, Segrin C, et al. Association of asthma illness representations and reported controller medication adherence among school-aged children and their parents. J Pediatr Health Care 2017;31:703-12.

39 Kosse RC, Koster ES, Kaptein AA, et al. Asthma control and quality of life in adolescents: the role of illness perceptions, medication beliefs, and adherence. J Asthma 2020;57:1145-54.

40 Tiggelman D, van de Ven MOM, van Schayck OCP, et al. Longitudinal associations between asthma control, medication adherence, and quality of life among adolescents: results from a cross-lagged analysis. Qual Life Res 2015;24:2067-74.

41 Juniper EF, Guyatt GH, Feeny DH, et al. Measuring quality of life in children with asthma. Qual Life Res 1996;5:35-46.

42 Juniper EF, Guyatt GH, Feeny DH, et al. Measuring quality of life in the parents of children with asthma. Qual Life Res 1996;5:27-34.

43 Epstein NB, Baldwin LM, Bishop DS. The McMaster family assessment DEVICE*. J Marital Fam Ther 1983;9:171-80.

44 Bihun JT, Wamboldt MZ, Gavin LA, et al. Can the family assessment device (FAD) be used with school aged children? Fam Process 2002;41:723-31.

45 Broadbent E, Petrie KJ, Main J, et al. The brief illness perception questionnaire. J Psychosom Res 2006;60:631-7.

46 Valero-Moreno S, Lacomba-Trejo L, Casaña-Granell S, et al. Psychometric properties of the questionnaire on threat perception of chronic illnesses in pediatric patients. Rev Lat Am Enfermagem 2020;28:e3242.

47 Horne R, Weinman J, Hankins M. The beliefs about medicines questionnaire: the development and evaluation of a new method for assessing the cognitive representation of medication. Psychol Health 1999;14:1-24.

48 Yilmaz O, Eroglu N, Ozalp D, et al. Beliefs about medications in asthmatic children presenting to emergency department and their parents. J Asthma 2012;49:282-7.

49 Weiner BJ, Lewis CC, Stanick C, et al. Psychometric assessment of three newly developed implementation outcome measures. Implement Sci 2017;12:108.

50 Brooke J. System usability scale usability.gov: improving the user experience: US. Department of Health \& Human Services, 2018. Available: https://www.usability.gov/how-to-and-tools/methods/ system-usability-scale.html [Accessed 17 Jun 2018].

51 Kenny DA, Cook W. Partner effects in relationship research: conceptual issues, analytic difficulties, and illustrations. Pers Relatsh 1999;6:433-48.

52 International Committee of Medical Journal Editors. Defining the role of authors and contributors, 2021. Available: http://www.icmje.org/ recommendations/browse/roles-and-responsibilities/defining-therole-of-authors-and-contributors.html [Accessed 20 Dec 2021]. 